\title{
The Subjective Consequences of Experiencing Random Events
}

\author{
Jason Hubbard $^{1}$, Tanaz Molapour ${ }^{2} \&$ Ezequiel Morsella ${ }^{3,4}$ \\ ${ }^{1}$ Department of Psychology, University of Oregon, Eugene, Oregon, USA \\ ${ }^{2}$ Department of Clinical Neuroscience, Karolinska Institutet, Stockholm, Sweden \\ ${ }^{3}$ Department of Psychology, San Francisco State University, San Francisco, CA, USA \\ ${ }^{4}$ Department of Neurology, University of California San Francisco, San Francisco, CA, USA \\ Correspondence: Jason Hubbard, Department of Psychology, University of Oregon, Eugene, OR, 97403, USA. \\ E-mail: hubbard3@uoregon.edu
}

Received: March 21, 2016

Accepted: April 14, 2016

Online Published: May 10, 2016

doi:10.5539/ijps.v8n2p120

URL: http://dx.doi.org/10.5539/ijps.v8n2p120

\begin{abstract}
In everyday life, one's experience is usually highly structured, coherent, and predictable, a regularity stemming from the many constraints (e.g., cultural and physical constraints) operating upon the natural and social worlds. Consider that events that are experienced in an office meeting are usually not experienced in the great outdoors, and vice versa. This predictability of the outside world is capitalized upon by the brain, which is highly prospective and incessantly extracts meaningful patterns from event sequences. Despite these considerations, to our knowledge there have been no investigations into the ways that the brain copes with experiences that violate this structured regularity. Here we demonstrate a novel paradigm designed to tax this prospective system (by presenting the brain with a rapid series of random events) and show that such exposure reliably induces negative affect. Participants are exposed to Rapid, Random Semantic Activation (RRSA) prior to completing a mood scale; compared to a mood baseline, RRSA yields a consistent pattern of negative affect. This pattern did not emerge in a control group that completed a task with identical stimuli. While previous research has focused on randomness in terms of humans' ability to produce and detect random sequences, our paradigm explores this issue as it relates to human experience. Our findings are consistent with the idea that, due to the prospective nature of the brain and one's "epistemic needs" (Kruglanski, 1980), gross violations in the regularity of experience produce some form of negative subjective experience.
\end{abstract}

Keywords: randomness, affect, consciousness, subjective

\section{Introduction}

The experiences that come one's way in everyday life are usually highly structured, coherent, and predictable, a regularity often stemming from the many physical and cultural constraints of the natural and social worlds. It is usually not the case that events experienced in, say, the great outdoors are also experienced during an office business meeting, or vice versa. Satisfying one's "epistemic needs" (Kruglanski, 1980), this regularity is capitalized upon by a highly prospective brain, one that, for adaptive purposes, incessantly extracts meaningful patterns from event sequences (Schacter \& Addis, 2007; Wolford et al., 2004). Given the statistical regularities of the outside world, and the brain's propensity towards capitalizing on those regularities in order to make predictions, questions remain regarding how the brain would respond to the absence of this regularity.

The topic of randomness has been thoroughly explored in the past (c.f., Lopes, 1982; Arrington \& Logan, 2004; Mayr \& Bell, 2006; Baddeley, 1966), but almost exclusively in the context of detecting or producing random sequences of events. Indeed, it has been demonstrated that people consistently deviate from randomness in these contexts. In the gambler's fallacy, when asked to rate the randomness of a sequence, people under-estimate long runs of the same event due to their use of the representative heuristic (Tversky \& Kahneman, 1974). When asked to behave in a random manner (e.g., randomly switching between two different tasks), people commit the opposite error, exhibiting a bias towards perseverating (Arrington \& Logan, 2004). These failures to behave randomly may be attributed to bottlenecks in processes such as response selection (Baddeley, 1966) or driven (at least in part) by stimulus-driven changes in the environment (Mayr \& Bell, 2006). Clearly, the previous work has demonstrated that humans have difficulty interpreting and producing random sequences, and has provided at least some mechanistic explanation of how this occurs. What the previous work has not addressed, however, is 
the broader question of why one would expect that humans should be able to intuitively comprehend randomness. Is there any evolutionary benefit to random thinking? In situations like a soccer or tennis match, production of random behavior confers some advantage, and it has been found that players do in fact approach random behavior in these contexts (Palacios-Huerta, 2003; Walker \& Wooders, 2001). Others would argue that the cognitive system is biased towards prediction and pattern extraction (Wolford et al., 2004), an ability that has been conserved due to the evolutionary advantages of being able to anticipate one's environment to utilize resources. So, while humans are capable of achieving randomness in some situations, this appears to run counter to a strong bias towards prospection.

The world around us can often be unpredictable, but despite this, there are regularities to our experience that follow naturally by virtue of the physical properties of our surroundings. For instance, we do not suddenly jump from one environment to another, but travel between them through a series of intermediate steps (e.g., by bike, car, airplane). Additionally, within each environment there are predictable clusters of objects, agents, and events that are more likely to occur together; while on a camping trip, one would be surprised to come across a photocopier, just as one would be equally surprised to encounter a bear while in a business meeting. While there is variability to our experience, that experience is clearly bounded by physical, cultural, and social constraints. These constraints are what make prediction possible, and enable us to thrive in complex environments.

Given that our subjective experience of the world is inherently constrained by the regularity of our environment, probing the concept of randomness using abstract sequences may fail to reveal the fundamental prospective processes of the brain. For instance, asking one to rate the randomness of a given sequence entails an implied question of agency and intention: "Was the agent who produced this sequence acting purely randomly, or according to some rule?" A system that is inclined to seek out and exploit regularity will be biased towards seeking out the convoluted pattern that underlies the sequence. Additionally, this system will be inclined towards using efficient heuristics based on previous knowledge to infer properties of the sequence (e.g., the representative heuristic). Thus, this method reduces to a question of inference, rather than random thinking per se. Even when considering random sequences of events, it has been suggested that some so-called fallacies (e.g., representativeness) are actually good predictors of real-world outcomes, assuming finite event sequences (Hahn $\&$ Warren, 2009). As infinite sequences are not consistent with our normal experience in the world, it is thus not surprising that we are poorly equipped to reason based on such assumptions, and simply enumerating the situations in which one fails to employ the correct problem-solving strategy does not offer much insight into the underlying cognitive processes involved (Gigerenzer, 1996). Rather than using abstract sequences it is perhaps more fruitful, then, to explore randomness in terms of subjective experience. An experience that is devoid of order, meaning, and predictability would run counter to the subjective status quo. The aim of the present project was to examine the boundary conditions with respect to the prospective nature of the brain. Specifically, we were interested in the subjective consequences of exposing the cognitive system to a situation in which prospection is impossible - a random experience. Since a random experience would not conform to the brain's natural proclivity towards order and meaning (Wolford et al., 2004; Kruglanski, 1980), many would predict that the event would yield subtle, negative changes in subjective experience.

In order to explore these questions, one could utilize a paradigm that somehow induces a random experience, while probing the perturbations in subjective experience that occur from such randomness. Accordingly, we envisioned an experiment in which the subject, before having his or her mood assessed, experienced a random sequence of events - first experiencing an event from a zoo visit, then one from a soccer match, and then one from giving a lecture, and so forth. Naturally, such an experiment is unfeasible. Hence, instead of using actual experiences as stimuli, we used the perceptuo-semantic properties of a single word as an instance of an "event" and had participants continually process the semantics of a rapid and random sequence of words. Words were chosen for this Rapid, Random Semantic Activation (RRSA) over other possible stimuli (e.g., images) for several reasons. In particular, it is difficult to produce a large body of images that are equivalent in their low-level perceptual properties (e.g., luminance, complexity) while also eliciting unambiguous meanings in each individual. Words are composed of a discrete set of familiar symbols and, as experimental stimuli, can have their distinct properties (e.g., word frequency, length, familiarity) taken into account. When randomized, the semantic meanings of these words form a string of "events" that do not conform to any interpretable narrative. A system designed to extract meaning from event sequences (Wolford et al., 2004) would struggle with processing such strings. By contrast, the perceptual system is capable of processing low-level properties of these same words (e.g., the color of the letters) with aplomb. In our paradigm, we capitalize on this asymmetry in processing either the perceptual or semantic features of words; by attending to the semantic content, we can approximate a random experience, while attending to the perceptual features of the same stimuli will not induce such an experience. 
While similar manipulations have been used extensively in the realm of learning and memory (Craik \& Lockhart, 1972), here we are interested in the subjective consequences of the different modes of processing. We used a between-subjects design in which one group of participants performed a lexical decision task (i.e., assessing whether a stimulus is a word or nonword) and a control group responded to the hue in which words were presented. Further, we used a rapid pace of stimulus presentation to induce the latter group to truly adopt a shallow processing strategy and not attend to the word meanings. To examine the subjective consequences of the Rapid, Random Semantic Activation (RRSA), we administered a brief mood scale prior to and immediately following the task. Given the previous evidence that the brain is inherently prospective, and that a random experience would run against the subjective status quo, we hypothesized that the RRSA condition would yield subtle, negative changes in mood, relative to the control group.

\section{Method}

A total of 54 students from the San Francisco State University subjects pool participated in exchange for course credit. To establish a mood baseline, participants first completed by hand a modified version of the Brief Mood Introspection Scale (BMIS; Mayer \& Gaschke, 1988). The scale consists of 16 adjectives (e.g., "lively", "sad") covering eight distinct moods, rated on a 4-point scale ( $1=$ "definitely do not feel" through $4=$ "definitely feel"). In our variant of the scale, we used 9 of the adjectives, which fell into three categories: Positive Affect (lively, happy, content, calm), Negative Affect (sad, gloomy, jittery, nervous), and Effort (tired). The scoring of Positive Affect and Negative Affect was based on the scoring of the Pleasantness and Negative scales on the full BMIS, respectively (Mayer \& Gaschke, 1988). Importantly, "Effort" was used to assess differences in task difficulty between conditions, to address the explanation that observed differences in mood are driven by task difficulty. To provide a more fine-grained analysis, participants rated each item on an 8-point (instead of 4-point) scale.

Participants were then randomly assigned to either the "Shallow" or "RRSA" condition. In both conditions, participants responded to a list of words, each presented for $700 \mathrm{~ms}$ in a particular color (red, green, or blue). Words $(n=1246)$ were gathered from the MRC Psycholinguistic Database (Colheart, 1981), selected on the basis of length (4-12 letters), frequency, imageability, concreteness, meaningfulness, and familiarity. Nonwords ( $n=139$; 4-9 letters in length), gathered from the ARC Nonword Database (Rastle, Harrington, \& Colheart, 2002), conformed to English syntactical and morphological rules. The entire list consisted of 1385 words, with $10 \%(n=139)$ being nonwords. In the Shallow condition, participants were instructed to respond by pressing the spacebar to any word (or nonword) presented in a particular color (red, green, or blue, target color counter-balanced across participants). In the RRSA condition, participants pressed the spacebar whenever they saw a nonword and disregarded color. The color of the word was randomly determined on each trial, and the response-relevant color was presented in only $10 \%$ of the trials. In the RRSA condition, where color was irrelevant, one color was presented only $10 \%$ of the time as well. The same items were presented randomly to each participant, regardless of condition, and each list elicited identical motor responses. Thus, the only difference between conditions was task instructions. Word presentation times $(700 \mathrm{~ms})$ were not self-paced to ensure that all participants saw each word for the same duration. The rapid pace of instruction also motivated participants in the Shallow condition to truly adopt a shallow processing strategy and only attend to the color of the words, as it would be difficult to attend to both stimulus dimensions in such a short amount of time. The total time to complete the task was approximately 15 minutes. After the block, participants immediately completed a post-measure of our mood scale.

\section{Results}

The mood scale consisted of nine items rated on an 8-point scale that fell into three dimensions: Positive Affect, Negative Affect, and Effort. For each participant $(n=54)$ these dimensions were calculated for both the pre-test and post-test measures, and the difference scores for the three dimensions were used in the analysis (Table 1).

Table 1. Mean change in mood score by condition

\begin{tabular}{llll}
\hline Condition & Positive Affect & Negative Affect & Effort \\
\hline RRSA & $-3.64 *(0.93)$ & $0.40^{*}(0.69)$ & $0.68(0.34)$ \\
Shallow & $-1.28(0.56)$ & $-1.28(0.45)$ & $0.92(0.32)$
\end{tabular}

$* \mathrm{p}<.05 ;$ Numbers in parentheses indicate SEM. 
Three participants were excluded due to failure to follow instructions and one participant was excluded due to computer error, leaving a total of 25 per condition. As predicted and revealed in Figure 1, RRSA led to an increase in the Negative Affect dimension compared to the Shallow condition, $t(48)=2.03, p=.048$ (Figure 1). An omnibus ANOVA with Rating Scale (Positive Affect, Negative Affect, and Effort) as one factor and Condition (RRSA vs. Shallow) as another factor revealed a significant interaction, $F(2,96)=4.68, p=.012$. Accordingly, those in the RRSA condition also decreased more in Positive Affect than those in the Shallow condition, $t(48)=2.17, p=.035$. Importantly, there was no difference in Effort, $t(48)=0.52, p=.608$, suggesting that differences in affect are not merely artifacts from the RRSA condition being more effortful than the shallow condition.

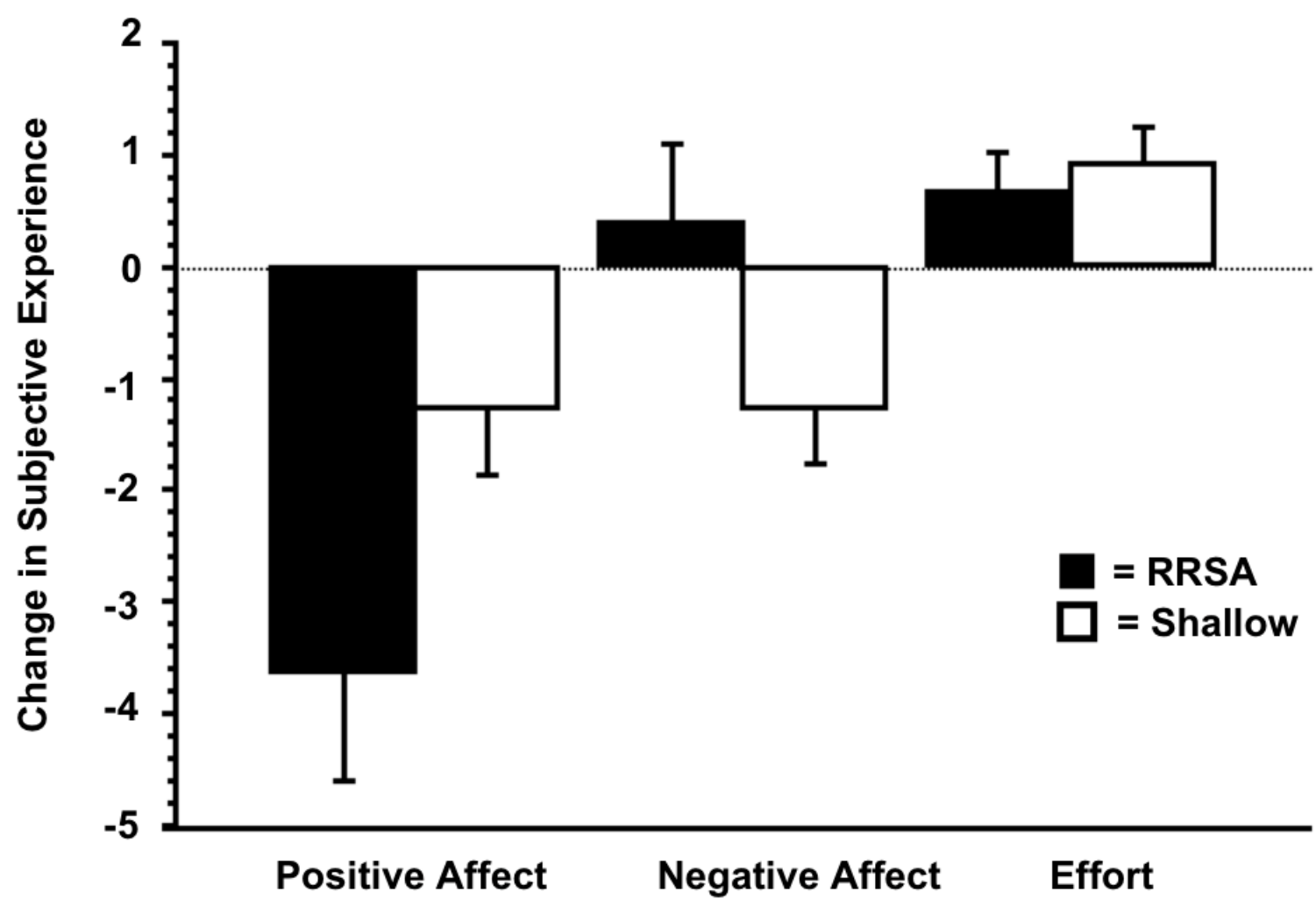

Figure 1. Change in subjective experience (positive affect, negative affect, and effort) following the Rapid Random Semantic Activation and Shallow conditions. Error bars indicate SEMs

The present study utilized a modified version of the original mood scale, which consisted of more items measured on a 4-point scale. Notably, the data here mirror the pattern seen in a pilot study $(n=27)$ using the same stimuli but utilizing the full version of the scale: it replicated the result for the Negative dimension, $t(25)=$ $2.95, p=.007$, as well as a trend regarding Pleasantness, $t(25)=1.87, p=.074$. While the difference in Effort in this pilot study approached significance $(t(25)=1.923, p=.066)$, those in the Shallow group reported more effort than RRSA, which is inconsistent with the hypothesis that the negative affect seen in RRSA is merely an artifact of being a more difficult task.

\section{Discussion}

The concept of randomness is not new to psychology or cognitive science. Indeed, investigations into the ability to detect or to produce random sequences of symbols (Lopes, 1982) or to randomly perform actions (Arrington \& Logan, 2004; Mayr \& Bell, 2006) have consistently found that randomness (in the mathematical sense) is a difficult concept for humans to understand. What has traditionally been ignored, however, is randomness as it relates to our subjective experience of the world. As we move throughout different environments, there are probabilistic regularities to the objects that surround us - while in the office, one frequently encounters staplers, copy machines, and desks, while walking down the street one can expect motorcycles, street lights, and signs. 
Furthermore, in order to change to another environment, one typically has to do so in several intervening steps (i.e., taking a walk, or travelling by bus or plane) and each step has its own clusters of objects and experiences associated with it. It is never the case that we instantaneously "jump" from one environment to another. Interestingly, the closest that one may have to such an experience involves complete loss of consciousness (e.g., from surgery).

In many cognitive realms (e.g., visual perception, semantic processing) the brain's use of expectation and pattern completion plays a significant role, and it is not unreasonable to expect that one's subjective experience in the world would be subserved by similar prospective mechanisms. The brain likely capitalizes on the statistical regularities of what it encounters, and develops to thrive in the predictive environments encountered in daily life, doing its best to make sense of what is occurring and predicting future events. It has been suggested that we have "epistemic needs" to have order and regularity in the world around us (Kruglanski, 1980). Indeed, it has been proposed that the structure of brain networks are fundamentally non-random and modular in nature (Sporns, 2011), and at a functional level, a general organizing principle of the brain is to minimize unpredictability between the external environment and internal states (Friston, Kilner, \& Harrison, 2006; Friston, 2012).

From the social, cognitive, and biological levels of analysis, the prospective nature of the brain appears to play a critical role. The aim of the present investigation was to begin to test the boundaries of these processes, to see how violations in the regularity of experience affect the system, and the subjective consequences of these violations. To our knowledge this is the first systematic investigation into the subjective effects of random experience. Consistent with our predictions and with existing theory, the experience of random events led to a reliable pattern of negative subjective experience. We believe this paradigm will prove to be a simple, yet powerful tool for exploring the fundamental prospective processes of the brain. These initial findings reveal a broad pattern of negative affect, but future work can reveal more fine-grained modulations in cognitive processes, and can easily be coupled with other dependent measures (e.g., imaging, psychophysiology). For instance, future work could examine to what extent these findings are specific to semantic activation-it is possible that similar subjective consequences arise from random sensory (visual or auditory) input as well. Other work could identify the locus of the negative affect, revealing whether it is a consequence of some active process attempting to make sense of the unstructured input, or a more passive consequence of the random activation of the semantic networks per se. Masking techniques could be utilized to examine whether conscious perception is necessary for these effects to occur, or whether subliminal random activation would also lead to consciously-experienced negative affect. A more difficult issue not addressed here is to examine the other side of the spectrum - namely, the minimal amount of structure in the input that aligns with the brain's prospective processes. This could be achieved using a hierarchically-organized semantic network and parametrically varying randomness by increasing the number of nodes that must be traversed in order to relate pairs of inputs. While there are a number of outstanding questions, we believe that the present findings are an important first step in revealing one of the brain's strongest affinities: the ability to comprehend and predict life's events.

\section{References}

Arrington, K. M., \& Logan, G. D. (2004). The cost of a voluntary task switch. Psychological Science, 15, 515-610. http://dx.doi.org/10.1111/j.0956-7976.2004.00728.x

Baddeley, A. D. (1966). The capacity for generating information by randomization. The Quarterly journal of experimental psychology, 18(2), 119-129. http://dx.doi.org/10.1080/14640746608400019

Berthoz, A. (2002). The brain's sense of movement. Harvard University Press, Cambridge.

Coltheart, M. (1981). The MRC Psycholinguistic Database. Quarterly Journal of Experimental Psychology, 33A, 497-505. http://dx.doi.org/10.1080/14640748108400805

Craik, F. I. M., \& Lockhart, R. S. (1972). Levels of Processing: A Framework for Memory Research. Journal of Verbal Learning and Verbal Behavior, 11, 671-684. http://dx.doi.org/10.1016/S0022-5371(72)80001-X

Friston, K. (2012). A Free Energy Principle for Biological Systems. Entropy, 14(12), 2100-2121. http://dx.doi.org/10.3390/e14112100

Friston, K., Kilner, J., \& Harrison, L. (2006). A free energy principle for the brain. Journal of Physiology-Paris, 100(1-3), 70-87. http://dx.doi.org/10.1016/j.jphysparis.2006.10.001

Gibson, J. J. (1979). The ecological approach to visual perception. Boston, MA: Houghton Mifflin.

Gigerenzer, G. (1996). On Narrow Norms and Vague Heuristics: A Reply to Kahneman and Tversky (1996). Psychological Review, 10(3), 592-596. http://dx.doi.org/10.1037/0033-295X.103.3.592 
Hahn, U., \& Warren, P. A. (2009). Perceptions of randomness: Why three heads are better than four. Psychological Review, 116(2), 454. http://dx.doi.org/10.1037/a0015241

Kruglanski, A. W. (1980). Lay epistemo-logic - Process and contents: Another look at attribution theory. Psychological Review, 87, 70-87. http://dx.doi.org/10.1037/0033-295X.87.1.70

Lopes, L. L. (1982). Doing the impossible: A note on induction and the experience of randomness. Journal of Experimental Psychology, Learning Memory and Cognition, 8, 626-636. http://dx.doi.org/10.1037/0278-7393.8.6.626

Mayer, J. D., \& Gaschke, Y. N. (1988). The experience and meta-experience of mood. Journal of Personality and Social Psychology, 55, 102-111. http://dx.doi.org/10.1037/0022-3514.55.1.102

Mayr, U., \& Bell, T. (2006). On how to be unpredictable: Evidence from the voluntary task-switching paradigm. Psychological Science, 17, 774-780. http://dx.doi.org/10.1111/j.1467-9280.2006.01781.x

Meyer, D. E., \& Schvaneveldt, R. W. (1971) Facilitation in recognizing pairs of words: Evidence of a dependence between retrieval operations. Journal of Experimental Psychology: General, 90, 227-234. http://dx.doi.org/10.1037/h0031564

Palacios-Huerta, I. (2003). Professionals play minimax. The Review of Economic Studies, 70(2), 395-415. http://dx.doi.org/10.1111/1467-937X.00249

Rastle, K., Harrington, J., \& Coltheart, M. (2002). The ARC Nonword Database. Quarterly Journal of Experimental Psychology, 55A, 1339-1362. http://dx.doi.org/10.1080/02724980244000099

Schacter, D. L., \& Addis, D. R. (2007). The cognitive neuroscience of constructive memory: Remembering the past and imagining the future. Philosophical Transactions of the Royal Society of London, Series B: Biological Sciences, 362, 773-786. http://dx.doi.org/10.1098/rstb.2007.2087

Sporns, O. (2011). The Non-Random Brain: Efficiency, Economy, and Complex Dynamics. Frontiers in computational neuroscience, 5, 5. http://dx.doi.org/10.3389/fncom.2011.00005

Tversky, A., \& Kahneman, D. (1974). Judgment under uncertainty: Heuristics and biases. Science, 185(4157), 1124-1131. http://dx.doi.org/10.1126/science.185.4157.1124

Walker, M., \& Wooders, J. (2001). Minimax play at Wimbledon. The American Economic Review, 91(5), 1521-1538. http://dx.doi.org/10.1257/aer.91.5.1521

Wolford, G., Miller, M. B., \& Gazzaniga, M. S. (2004). Split Decisions. In M. S. Gazzaniga (Ed.), The Cognitive Neurosciences III (pp. 1189-1199). Cambridge, MA: MIT Press.

\section{Copyrights}

Copyright for this article is retained by the author(s), with first publication rights granted to the journal.

This is an open-access article distributed under the terms and conditions of the Creative Commons Attribution license (http://creativecommons.org/licenses/by/3.0/). 